\title{
Numerical analysis of MHD casson fluid flow over an exponentially accelerated vertical plate in embedded porous medium with ramped wall temperature and ramped surface concentration in uniform magnetic field
}

\author{
Ch. Vijaya Bhaskar', Siva Reddy Sheri', Anjan Kumar Suram ${ }^{3}$ \\ ${ }^{1}$ Department of Mathematics, Vignan Institute of Technology and Science, India \\ ${ }^{2}$ Department of Mathematics, GITAM University, India \\ ${ }^{3}$ Department of Mathematics, Christu Jyothi Institute of Technology and Science, India
}

\begin{abstract}
Article Info
Article history:

Received Jun 27, 2019

Revised Oct 22, 2019

Accepted Feb 19, 2020

Keywords:

Casson fluid

FEM

MHD

ABSTRACT

Numerical analysis of MHD casson fluid flow over an exponentially accelerated vertical plate in embedded porous medium with ramped wall temperature and ramped surface concentration has investigated in the current investigation. The flow governing dimensional velocity, temperature and concentration differential equations are converted into non dimensional form by using non-dimensional variables. Numerical solutions to the converted equations are obtained by finite element method. The results are presented graphically and in tabular form for various controlling parameters. In order to highlight the validity and accuracy of our present method, we have compared our results with the results obtained earlier. A very good validation of the present numerical results has been achieved.
\end{abstract}

Ramped temperature

Thermal radiation

\section{Corresponding Author:}

Ch. Vijaya Bhaskar,

Department of Mathematics,

Vignan Institute of Technology and Science, Hyderabad, Telangana, India.

Email: vijay3284@gmail.com
This is an open access article under the CC BY-SA license.

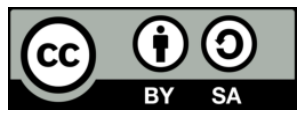

\section{INTRODUCTION}

Magnetohydrodynamics (MHD) is the study of the interaction between magnetic fields and electrically moving conducting fluids. It came into existence in the late 1930s or early 1940s. Later in nineteenth-century physicist Faraday conducted few isolated experiments on MHD. Afterwards astrophysicists realized how omnipresent magnetic fields and plasmas are throughout the universe. Then in 1942 invention Alfven waves created a significant attention in astrophysics. However, there was some early pioneering work by engineer J. Hartmann, who invented the electromagnetic pump in 1918. Hartmann also undertook a systematic theoretical and experimental investigation of the flow of mercury in a homogeneous magnetic field. Indeed the term 'Hartmann flow' is now used to describe duct flows in the presence of a magnetic field. Despite Hartmann's early researches, it was only in the early 1960s that MHD began to be exploited in engineering. However, as the interest in power generation declined, research into metallurgical MHD took off. Two decades later, magnetic fields are routinely used to heat, pump, stir and levitate liquid metals in the metallurgical industries. The key point is that the Lorentz force provides a non-intrusive means of controlling the flow of metals. With constant commercial pressure to produce cheaper, better and more consistent materials, MHD provides a unique means of exercising greater control over casting and refining processes. 
The MHD flows are encountered in many geophysical, astrophysical and engineering applications. Hydromagnetic flows have a key role in the fields of aeronautics, stellar and planetary magnetospheres. MHD concepts are utilized by the engineers in the design of heat exchangers, pumps, thermal protection, in space vehicle propulsion control and re-entry, in creating novel power-generating systems. The purification of molten metal from non-metallic inclusions through the application of magnetic field is another important feature of MHD. All such applications of MHD give rise to investigate the problems which involves the MHD effects. For instance, Nadeem et al. [1] have studied MHD flow of a casson fluid over an exponentially shrinking sheet. Shehzad et al. [2] have analyzed the effects of mass transfer on MHD flow of casson fluid with chemical reaction and suction. Casson et al. [3] have investigated Effects of radiation on MHD free convection of a casson fluid from a horizontal circular cylinder with partial slip in non-Darcy porous medium with viscous dissipation. Benazir et al. [4] have discussed unsteady MHD casson fluid flow over a vertical cone and flat plate with non-uniform heat source/sink. Effects of fluid suction on an oscillatory MHD channel flow with heat transfer studied by Ahmed et al. [5]. Effects of hall current on MHD slip flow of casson nanofluid over a stretching sheet with zero nanoparticle mass flux studied by Aziz and Afify [6].

A shear thinning liquid which is assumed to have an infinite viscosity at zero rate of shear, a yield stress below which no flow occurs, and a zero viscosity at an infinite rate of shear is known as casson fluid. The casson fluid is a plastic fluid, which yields shear stress in constitutive equations. Some of the examples of casson fluid model are jelly, soup, honey, tomato sauce, concentrated fruit juices, drilling operations, food processing, metallurgy, paints, coal in water, synthetic lubricants, manufacturing of pharmaceutical products, synovial fluids, sewage sludge, and many others. Human blood is also considered as casson fluid. The nonlinear casson constitutive equation described the properties of many polymers. Furthermore, casson fluid possesses yield stress and has great importance in polymer processing industries and biomechanics. Hari and Harshad [7] have described Soret and heat generation effects on MHD casson fluid flow past an oscillating vertical plate embedded through porous medium. Mohyuddin and Khan [8] have studied nonlinear radiation effects on squeezing flow of a casson fluid between parallel disks. Akbar [9] has analyzed the influence of magnetic field on peristaltic flow ofa casson fluid in an asymmetric channel: application in crude oil refinement. Vajravelu et al. [10] have presented peristaltic transport of a casson fluid in contact with a Newtonian fluid in a circular tube with permeable wall. Nagarani et al. [11] have given exact analysis of unsteady convective diffusion in casson fluid flow in an annulus-Application to catheterized artery. A mathematical modeling for pulsatile flow of casson fluid along with magnetic nanoparticles in a stenosed artery under external magnetic field and body acceleration discussed by Priyadharshini and Ponalagusamy [12].

Thermal radiation is electromagnetic radiation emitted by a body by virtue of its temperature and at the expense of its internal energy. Thus thermal radiation is of the same nature as visible light, $\mathrm{x}$-rays, and radio waves, the difference between them being in their wavelengths and the source of generation. Thermal radiation is important has applications that include high-temperature manufacturing processes and materials processing, improved efficiency, and more accurate design methods necessary for energy conversion devices, the use of new materials, hypersonic flow analysis, and others. Sajid and Hayat [13] have investigated the influence of thermal radiation on the boundary layer flow due to an exponentially stretching sheet. Bhattacharyya and Layek [14] have studied the effects of suction/blowing on steady boundary layer stagnation-point flow and heat transfer towards a shrinking sheet with thermal radiation. Pramanik [15] has explained casson fluid flow and heat transfer past an exponentially porous stretching surface in presence of thermal radiation. Effects of nonlinear thermal radiation on MHD chemically reacting Maxwell fluid flow past a linearly stretching sheet investigated by Mohan Rami Reddy et al. [16]. Yao-mathematical and numerical analysis of radiative heat transfer in semi-transparent media given by Chuang Han et al. [17].

Rate of change in temperature over time is called ramped temperature. In few investigations researchers considered ramped temperature profiles, the interval for ramped with respect to time in the plate temperature is assumed fixed i.e. $0<t^{\prime} \leq t_{0}\left(t^{\prime}\right.$ and $t_{0}$ are time and characteristic time respectively) which reduces to $0<t \leq 1$ ( $t$ being the dimensionless time) in non-dimensional form. It is to be noted that interval for ramped profile varies from material to material depending upon the specific heat capacity of the material. Variable ramped temperature profiles appear in real world situation in building air conditioning systems, fabrication of thin-film photovoltaic devices, phase transition in processing of materials, turbine blade heat transfer, heat exchangers etc. Siva Reddy Sheri et al. [18] have analyzed heat and mass transfer effects on MHD natural convection flow past an impulsively moving vertical plate with ramped temperature. Siva Reddy Sheri and Anjan Kumar [19] have discussed finite element analysis of heat and mass transfer past an impulsively moving vertical plate with ramped temperature. Siva Reddy Sheri et al. [20] have investigated heat and mass transfer effects on MHD natural convection flow past an infinite inclined plate with ramped temperature. Nandkeolyar et al. [21] have given exact solutions of unsteady MHD free convection in a heat absorbing 
fluid flow past a flat plate with ramped wall temperature. Seth et al. [22] have clarified hydromagnetic natural convection flow with heat and mass transfer of a chemically reacting and heat absorbing fluid past an accelerated moving vertical plate with ramped temperature and ramped surface concentration through a porous medium. MHD Natural convections flow past a moving vertical plate with ramped temperature studied by Vijaya Bhaskar et al. [23].

As per authors knowledge no work has taken place to investigate the numerical analysis on MHD casson fluid flow over an exponentially accelerated vertical plate in embedded porous medium with ramped wall temperature and ramped surface concentration. In the current numerical investigation the influence of casson parameter and thermal radiation on hydromagnetic, incompressible, viscous electrically conducting, heat absorbing fluid over an exponentially accelerated vertical plate in embedded porous medium with ramped temperature and ramped surface concentration has been studied. The flow governing partial differential equations along with initial and boundary conditions are transformed into non-dimensional form then solved with the aid of finite element method (FEM). The effects of various pertinent parameters on flow pattern are displayed graphically and interpreted in detail. Verification of computations with previous studies in the literature is also included. The results with respect to dimensionless velocity, temperature, and concentration can be used as a reference for any future study.

\section{FORMULATION OF THE PROBLEM}

In Figure 1 the flow being confined to $y^{\prime}>0$, where $y^{\prime}$ the coordinate is is measured in the normal direction to the plate and $x^{\prime}$-is taken along the wall in the upward direction. Initially, at time $t^{\prime}=0$, both the fluid and the plate are uniform temperature $T_{\infty}{ }^{\prime}$ and the concentration near the plate is assumed to be $C_{\infty}{ }^{\prime}$ at all the points respectively. At time $t^{\prime}>0$, the plate is given an exponentially accelerated in the vertical direction against gravitational field with velocity $U_{0} e^{a^{\prime} t}$ and constant heat flux ${ }_{T_{\infty}}{ }^{\prime}+\left(T_{w}{ }^{\prime}{ }^{+} T_{\infty}{ }^{\prime}\right) \frac{t^{\prime}}{t_{0}}$, when $t^{\prime} \leq t_{0}$ and $T_{w}{ }^{\prime}$ when $t^{\prime}>t_{0}$ respectively which is there after maintained constant $T_{w}{ }^{\prime}$ and the level of mass transfer at

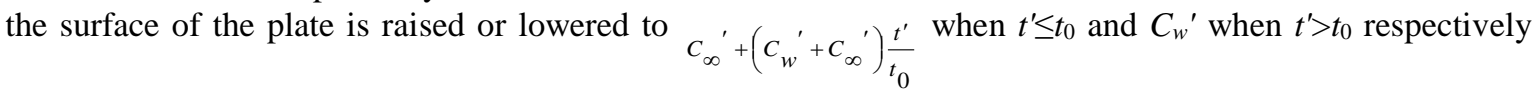
which is there after maintained constant $C_{w}{ }^{\prime}$. A uniformly magnetic field of strength $B_{0}$ is applied in the $y^{\prime}$-axis direction. Induced magnetic field is negligible. We assume that, rigid plate, incompressible flow, one dimensional flow, non-Newtonian fluid, free convection and viscous dissipation term in the energy equation is neglected.

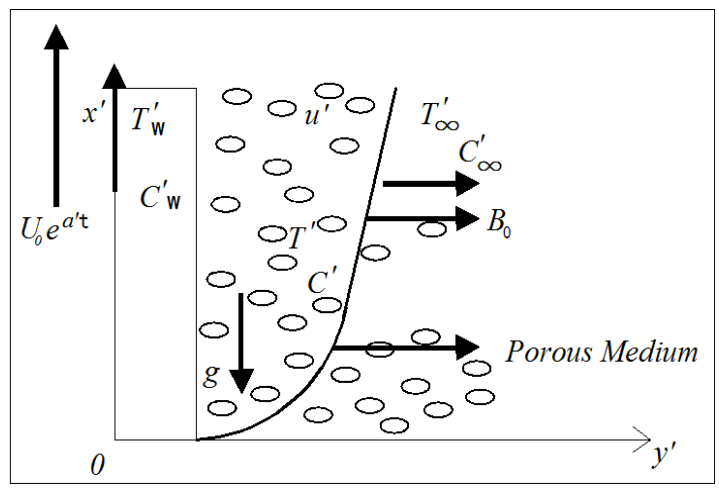

Figure 1. Sketch of the physical flow problem

The constitutive equation for the casson fluid can be written as:

$$
\tau_{i j}=\left\{\begin{array}{ll}
2\left(\mu B+\frac{P y}{\sqrt{2 \pi}}\right) e_{i j} & \pi>\pi_{c} \\
2\left(\mu B+\frac{P y}{\sqrt{2 \pi}}\right) e_{i j} & \pi<\pi_{c}
\end{array}\right\}
$$


where $\pi=e_{i j} e_{i j}$ and $e_{i j}$ is the $(i, j)$ th component of the deformation rate, $\pi$ is the product of the component of deformation rate with itself, $\pi_{c}$ is a critical value of this product based on the non-Newtonian model, $\mu \mathrm{B}$ is plastic dynamic viscosity of the non-Newtonian fluid and $P y$ is yield stress of fluid. Under above assumptions and taking into account the Boussinesq approximation, governing equations are given in (2-4).

$$
\begin{aligned}
& \rho \frac{\partial u^{\prime}}{\partial t^{\prime}}=\mu B\left(1+\frac{1}{\gamma}\right) \frac{\partial^{2} u^{\prime}}{\partial y^{\prime 2}}-\sigma B_{0}{ }^{2} u^{\prime}-\frac{\mu \phi}{k_{1}^{\prime}} u^{\prime}+\rho g \beta_{T}{ }^{\prime}\left(T^{\prime}-T_{\infty}^{\prime}\right)+\rho g \beta_{C}{ }^{\prime}\left(C^{\prime}-C_{\infty}^{\prime}\right) \\
& \frac{\partial T^{\prime}}{\partial t^{\prime}}=\frac{k}{\rho c p} \frac{\partial^{2} T^{\prime}}{\partial y^{\prime 2}}+\frac{1}{\rho c_{p}} \frac{\partial q^{\prime} r}{\partial y^{\prime}}+\frac{Q_{0}}{\rho c_{p}}\left(T^{\prime}-T_{\infty}^{\prime}\right) \\
& \frac{\partial C^{\prime}}{\partial t^{\prime}}=D_{M} \frac{\partial^{2} C^{\prime}}{\partial y^{\prime 2}}-k_{2}{ }^{\prime}\left(C^{\prime}-C_{\infty}^{\prime}\right)
\end{aligned}
$$

With following initial and boundary conditions:

$$
\left.\begin{array}{rl}
u^{\prime} & =0, T^{\prime}=T_{\infty}^{\prime}, C^{\prime}=C_{\infty}^{\prime} \text { for } y^{\prime} \geq 0 \text { and } t^{\prime} \leq 0, u^{\prime}=U_{0} e^{a^{\prime} t}, \text { at } y^{\prime}=0 \text { for } t^{\prime}>0, \\
T^{\prime} & =\left\{\begin{array}{l}
T_{\infty}^{\prime}+\left(T_{w}^{\prime}-T_{\infty}^{\prime}\right) t^{\prime} / t_{0} \text { at } y^{\prime}=0 \text { for } 0<t^{\prime}<t_{0}, \\
T_{w}^{\prime} \text { at } y^{\prime}=0 \text { for } t^{\prime}>t_{0},
\end{array}\right\} \\
C & =\left\{\begin{array}{l}
C_{\infty}^{\prime}+\left(C_{w}^{\prime}+C_{\infty}^{\prime}\right) t^{\prime} / t_{0} \text { at } y^{\prime}=0 \text { for } 0<t^{\prime}<t_{0}, \\
C_{w}^{\prime} \text { at } y^{\prime}=0 \text { for } t^{\prime}>t_{0},
\end{array}\right\} \\
u^{\prime} \rightarrow 0, T^{\prime} \rightarrow T_{\infty}^{\prime}, \quad C^{\prime} \rightarrow C_{\infty}^{\prime} \text { as } y^{\prime} \rightarrow \infty \text { for } t^{\prime} \geq 0
\end{array}\right\}
$$

Introducing the following dimensionless quantities:

$$
\begin{aligned}
& y=\frac{y^{\prime}}{U_{0} t_{0}}, u=\frac{u^{\prime}}{U_{0}}, t=\frac{t^{\prime}}{t_{0}}, \theta=\frac{\left(T^{\prime}-T_{m}^{\prime}\right)}{\left(T_{w}^{\prime}-T_{w}^{\prime}\right)}, C=\frac{\left(C^{\prime}-C_{\infty}^{\prime}\right)}{\left(C_{w}^{\prime}-C_{w}^{\prime}\right)}, G=\frac{\nu g / \beta_{j}^{\prime}\left(T_{w}^{\prime}-T_{m}^{\prime}\right)}{U_{0}^{3}}, G m=\frac{\nu g / \beta_{1}^{\prime}\left(C_{w}^{\prime}-C_{w}^{\prime}\right)}{U_{0}^{3}},
\end{aligned}
$$

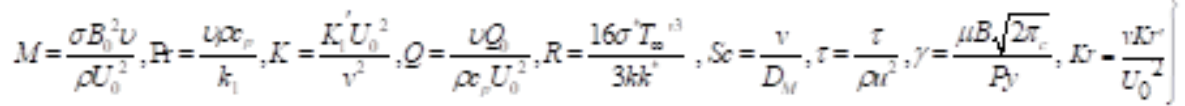

Making use of (6) in (2), (3), and (4), we get non-dimensional form as:

$$
\begin{aligned}
& \frac{\partial u}{\partial t}=\left(1+\frac{1}{\gamma}\right) \frac{\partial^{2} u}{\partial y^{2}}-\left(M+\frac{1}{K}\right) u+G r \theta+G m C \\
& \frac{\partial T}{\partial t}=\frac{1}{\operatorname{Pr}} \frac{\partial^{2} T}{\partial y^{2}}+(Q+R) T \\
& \frac{\partial C}{\partial t}=\frac{1}{S c} \frac{\partial^{2} C}{\partial y^{2}}-K r C
\end{aligned}
$$

The non-dimensional form of initial and boundary conditions are:

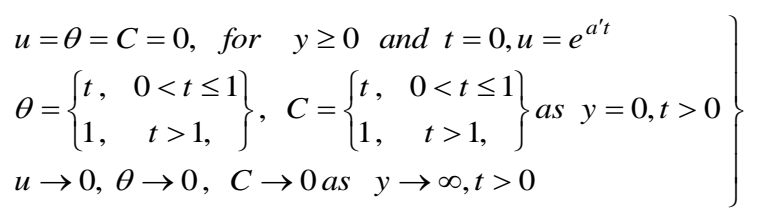




\section{METHOD OF SOLUTION}

The finite element method is a powerful technique for solving differential or partial differential equations as well as integral equations. An excellent description of finite element formulations is available in Bathe [24] and Reddy [25]. The basic concept is that the whole domain is divided into smaller elements of finite dimensions called "Finite Elements". It is the most versatile numerical technique in modern engineering analysis and has been employed to study various problems in heat transfer, fluid mechanics, chemical processing, rigid body dynamics, solid mechanics, electrical systems and many other fields. The finite element method (FEM) consists of following steps:

Step 1: Discretization of the domain

The fundamental concept of the FEM is to divide the region of the problem into small connected pieces, called finite elements. The group of elements is called the finite element mesh. These finite elements are associated in a non-overlapping manner, such that they completely cover the entire space of the problem. Step 2: Invention of the element equation

A representative element is selected from the mesh and the variational formulation of the given problem is created over the typical element. Over an element, an approximate solution of the variational problem is invented, and by surrogating this in the system, the element equations are generated. The element matrix, which is also known as stiffness matrix, is erected by using the element interpolation functions.

Step 3: Assembly of the element equations

The algebraic equations so achieved are assembled by imposing the inter element continuity conditions. This yields a large number of mathematical equations known as the global finite element model, which governs the whole domain.

Step 4: Imposition of the boundary conditions

On the accumulated equations, the initial and boundary conditions (10) are imposed.

Step 5: Solution of assembled equations

The assembled equations so obtained can be solved by any of the numerical methods, namely, Gauss elimination technique, LU decomposition technique, and the final matrix equation can be solved by iterative technique. For computational purposes, the coordinate y is varied from 0 to $y_{\max }=1.5$, where $y_{\max }$ represents infinity i.e., external to the momentum, energy and concentration edge layers.

\section{VALIDATIONS OF NUMERICAL RESULTS}

In order to validate and verify the present numerical method used for this study in the special case is compared with available results of Raj Nandkeolyar et al. [21] and Seth et al. [22] is exhibited in Tables 1-2. These tables display the comparison of Nusselt number and Sherwood number for both ramped and isothermal plates and it is conveyed there is good agreement between the results exist.

\begin{tabular}{|c|c|c|c|c|c|}
\hline \multirow[b]{2}{*}{$t$} & \multirow[b]{2}{*}{$\operatorname{Pr}$} & \multicolumn{2}{|c|}{ Results by Nandkeolyar et al. [21] } & \multicolumn{2}{|c|}{ Present Results } \\
\hline & & Ramped & Isothermal & Ramped & Isothermal \\
\hline 0.3 & 0.71 & 0.57134752 & 1.11605411 & 0.571347519 & 1.11605410 \\
\hline 0.7 & 0.71 & 0.96929143 & 0.92531051 & 0.969291430 & 0.92531050 \\
\hline 0.7 & 7.00 & 3.04350641 & 2.90540943 & 3.043506410 & 2.905409429 \\
\hline
\end{tabular}

Table 2. Comparison of Sherwood number (when $\gamma=\infty$ and $R=0$ )

\begin{tabular}{cccccc}
\hline & & \multicolumn{2}{c}{ Results by Seth et al. [22] } & \multicolumn{2}{c}{ Present Results } \\
\cline { 3 - 6 }$t$ & $K r$ & Ramped & Isothermal & Ramped & Isothermal \\
\hline 0.3 & 0.2 & 0.295649 & 0.525702 & 0.2956500 & 0.5257020 \\
0.5 & 0.2 & 0.386593 & 0.428415 & 0.3865929 & 0.4284149 \\
0.3 & 2.0 & 0.344659 & 0.839945 & 0.3446600 & 0.8399449 \\
\hline
\end{tabular}

\section{RESULTS AND DISCUSSION}

Extensive numerical solutions have been found for flow governing velocity, temperature and concentration (6-9) under the initial and boundary conditions (10), by using finite element method. Obtained computational results are presented graphically in Figures 2-16 to illustrate the influence of governing parameters viz. Cassion parameter, Magnetic parameter, porosity parameter, thermal Grashof number, mass Grashof number, Prandtl number, heat absorption, thermal radiation, Schmidt number and chemical reaction parameter on velocity, temperature and concentration. 
The behavior of fluid velocity against Cassion parameter $\gamma$ for both ramped and isothermal plates is illustrated in Figure 2. It reveals that an increase in $\gamma$ leads to diminish in velocity profile, since casson parameter makes the velocity boundary layer thickness shorter. It is further observed from this Figure 2 that plastic dynamic viscosity enlarged on increasing $\gamma$ it creates resistance in fluid flow and hence fluid velocity is decreased. Figure 3 depicts the influence of magnetic parameter $M$ on velocity profile for both ramped and isothermal plates. It is perceived from this Figure 3 that velocity gets reduce by increasing $M$. This is due to the fact that the applied magnetic field normal to the flow direction induces the drag like force called Lorentz force which has a tendency to decelerate fluid flow.

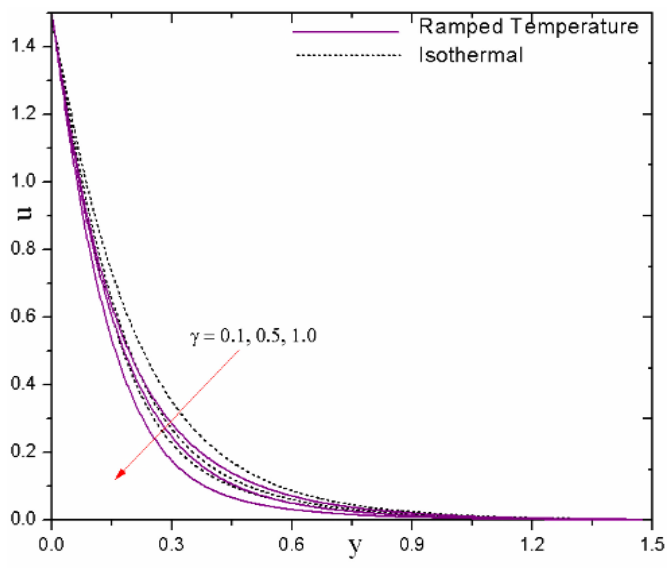

Figure 2. Velocity profile for casson parameter

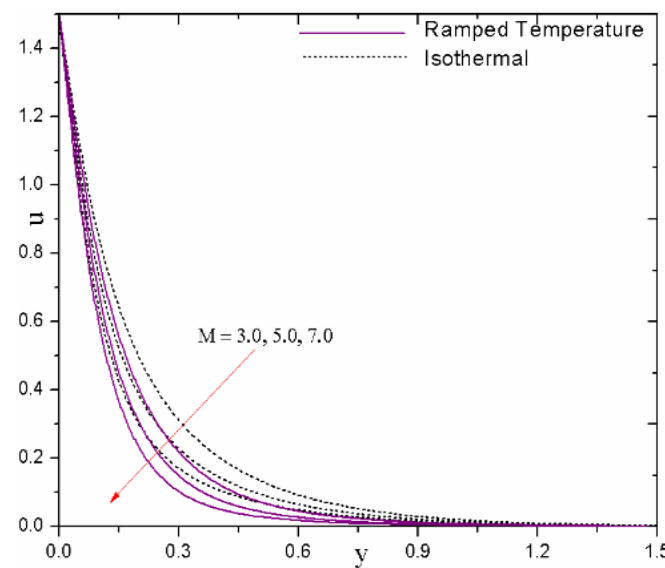

Figure 3. Velocity profile for magnetic parameter

In Figure 4 profiles of velocity have been plotted against various values of permeability parameter $K$ for both ramped and isothermal plates by keeping other parameters fixed. It is identified from this Figure 4 that for large values of $K$, velocity boundary layer thickness enlarges which explains the physical situation that as $K$ increases, the resistance of the porous medium is lowered which boosts up the momentum development of the flow regime, ultimately enhances the velocity field. The control of thermal Grashof number $\mathrm{Gr}$ on fluid velocity for both ramped and isothermal plates is demonstrated in Figure 5. Thermal Grashof number is the ratio of thermal buoyancy force to viscous hydro dynamic force. It is observed that velocity increases with increasing values of $G r$. The flow is accelerated due to the enhancement in the buoyancy forces corresponding to the increasing values of Grashof number.

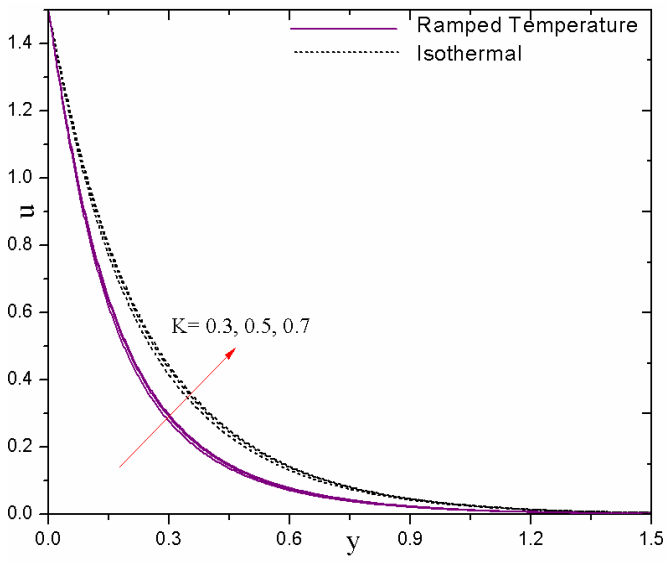

Figure 4. Velocity profile for porosity parameter

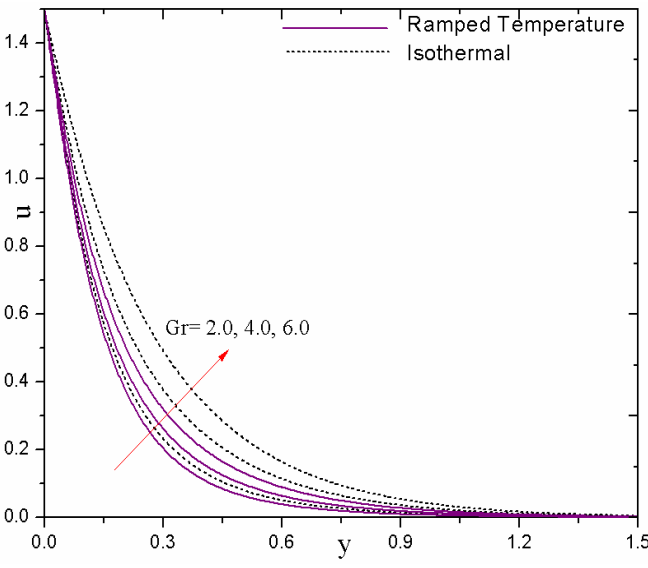

Figure 5. Velocity profile for Grashof number 
The influence of mass Grashof number on velocity profile for both ramped temperature and isothermal plate is displayed in Figure 6. The mass Grashof number $\mathrm{Gm}$ characterizes the ratio of the mass buoyancy force and viscous hydrodynamic force. As expected, it is observed that, for both ramped temperature and isothermal plate fluid velocity increases on increasing $\mathrm{Gm}$. This implies that fluid velocity is getting accelerated due to enrichment in mass buoyancy force. Figures 7-8 portray the effect $\mathrm{f}$ Prandtl number $\operatorname{Pr}$ on velocity and temperature profiles for both ramped temperature and isothermal plates. The Prandtl Number is a dimensionless number approximating the ratio of momentum diffusivity (kinematic viscosity) to thermal diffusivity.

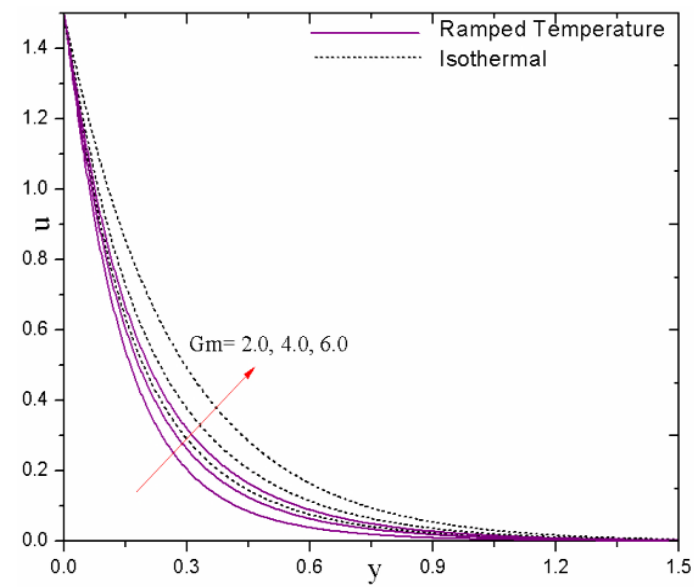

Figure 6. Velocity profile for mass Grashof number

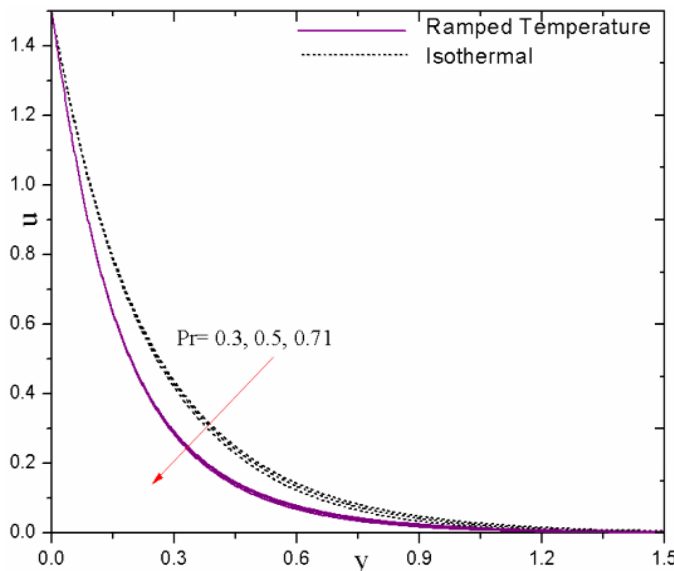

Figure 7. Velocity profile for Prandtl number

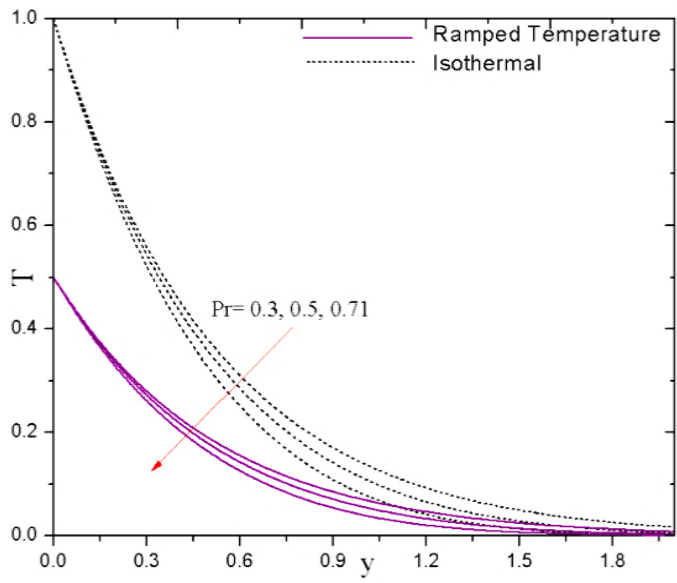

Figure 8. Temperature profile for Prandtl number

From Figure 7 it is perceived that velocity profile diminishes on rising Pr. Increases in Prandtl number significantly depress velocity boundary layer. Since Prandtl number causes an increase in the viscosity of the fluid which makes the fluid thick and it leads to a decrease in the velocity. Figure 8 reveals that temperature profile decreases on increasing Prandtl number. Physically, if $\operatorname{Pr}$ increases, the thermal diffusivity decreases and this phenomenon lead to decrease energy ability, which reduces the thermal boundary layer. The influence of the heat absorption $Q$ on the velocity and temperature fields for both ramped temperature and isothermal plates is presented in Figures 9 and 10. The presence of heat absorption boundary layer generates energy which cases the temperature of the fluid to increase. This increasing temperature produces an increase in the flow field due to buoyancy effect. 


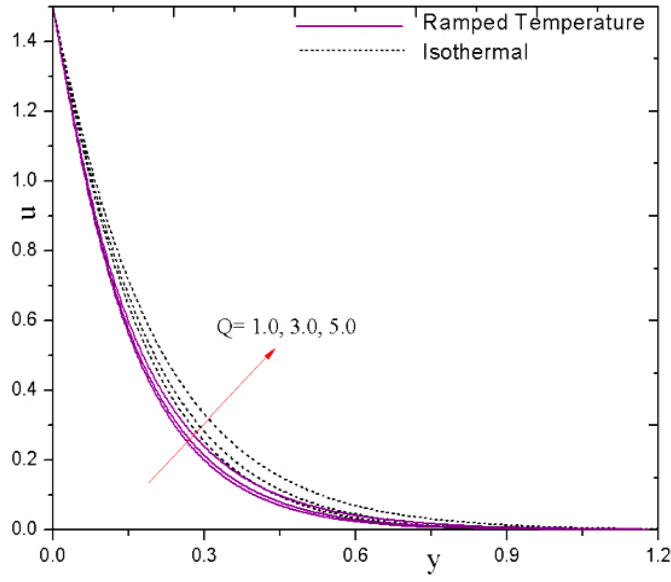

Figure 9. Velocity profile for heat absorption

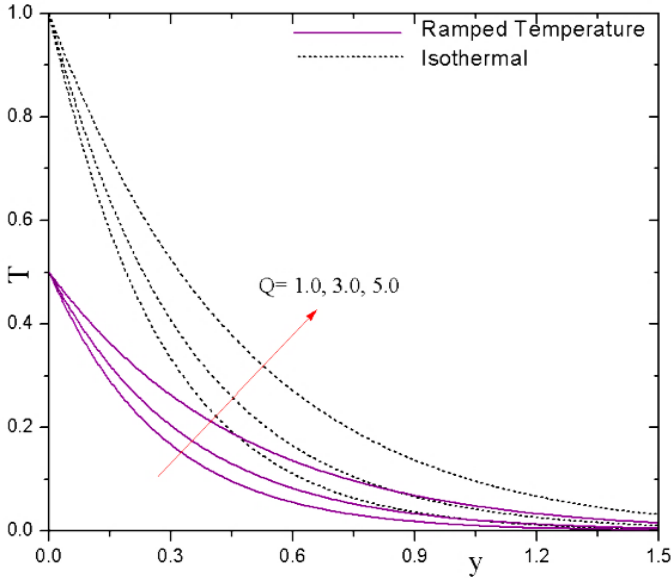

Figure10. Temperature profile for heat absorption

Velocity and temperature profiles against thermal radiation for both ramped and isothermal plates are displayed in Figures 11-12. From Figure 11 it is seen that an increase in radiation parameter $R$ leads to an increase in the fluid velocity for both ramped temperature and isothermal plates. Because when the strength of heat generated through thermal radiation is increased, the bond holding the components of the fluid particles is easily broken and the fluid velocity is increased. It is recognized from Figure 12 that thermal boundary layer is enhanced on increasing thermal radiation parameter $R$, it is due to the reason that thermal radiation has a tendency to enlarge the temperature profile for both ramped and isothermal plates.

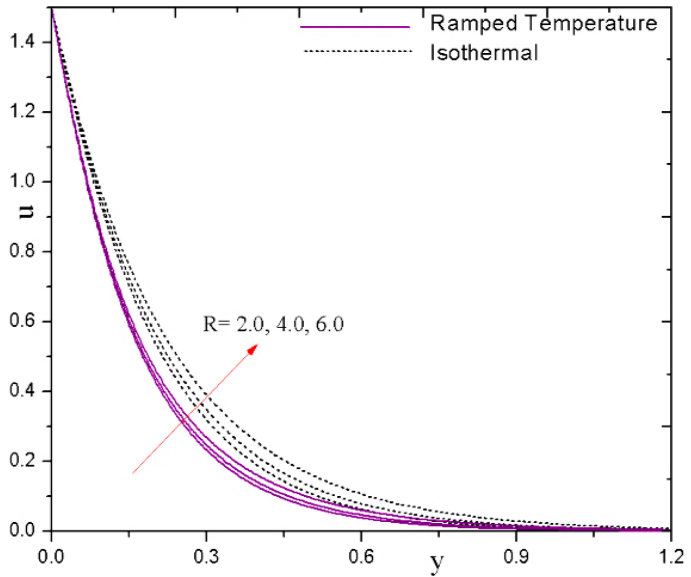

Figure 11. Velocity profile for thermal radiation

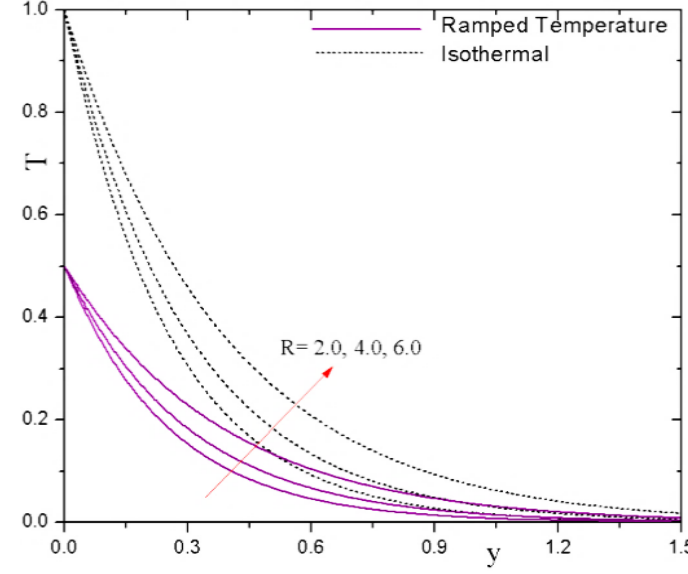

Figure 12. Temperature profile for thermal radiation

Figures 13-14 depict the influence of Schmidt number on the field of velocity and concentration. Schmidt number is a dimensionless number defined as the ratio of momentum diffusivity (viscosity) and mass diffusivity, and is used to characterize fluid flows in which there are simultaneous momentum and mass diffusion convection processes. As the Schmidt number increases, the concentration decreases. This causes the concentration buoyancy effects to decrease yielding a reduction in the fluid velocity. Reductions in the velocity and concentration distributions are accompanied by simultaneous reductions in the velocity and concentration boundary layers. 


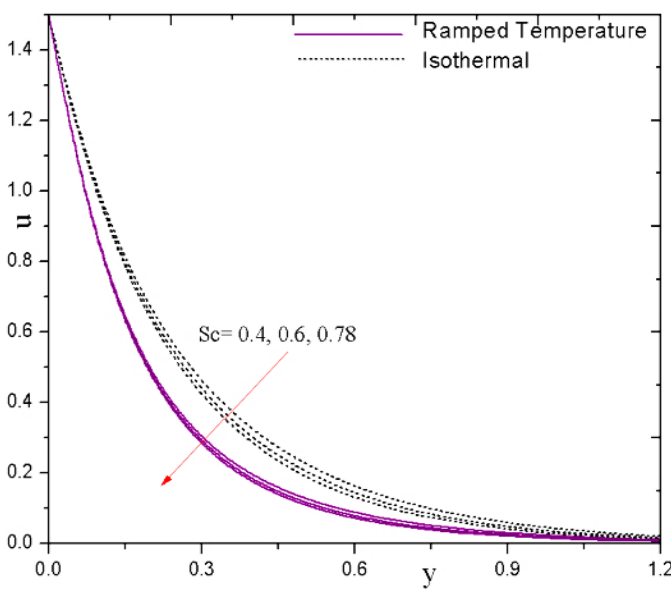

Figure 13. Velocity profile for Schmidt number

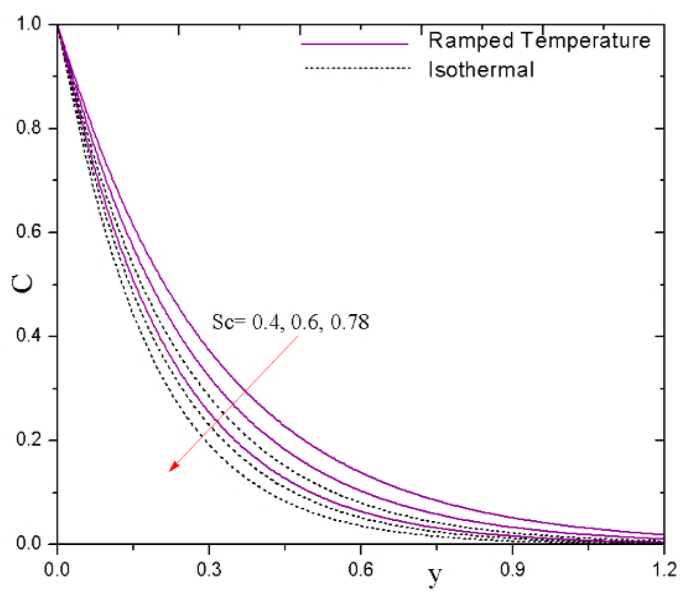

Figure 14. Concentration profile for Schmidt number

Effect of chemical reaction parameter on fluid velocity and concentration for both ramped and isothermal plates is presented in Figures 15-16. It is evident from Figure 15 that velocity profiles reduce on rising $K r$. This is due to the fact that the chemical reaction parameter has a retarding influence on the solute concentration which makes a decrease in the fluid velocity due to a reduced mass buoyancy force. Figure 16 reveals that an increase in chemical reaction parameter causes a decrease in concentration profile. It may be attributed that chemical reaction parameter retards concentration boundary-layer thickness.

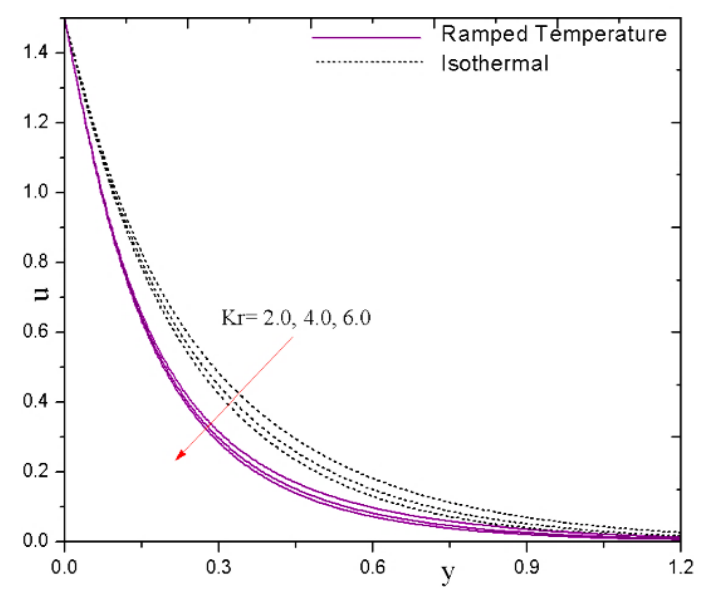

Figure 15. Velocity profile for chemical reaction

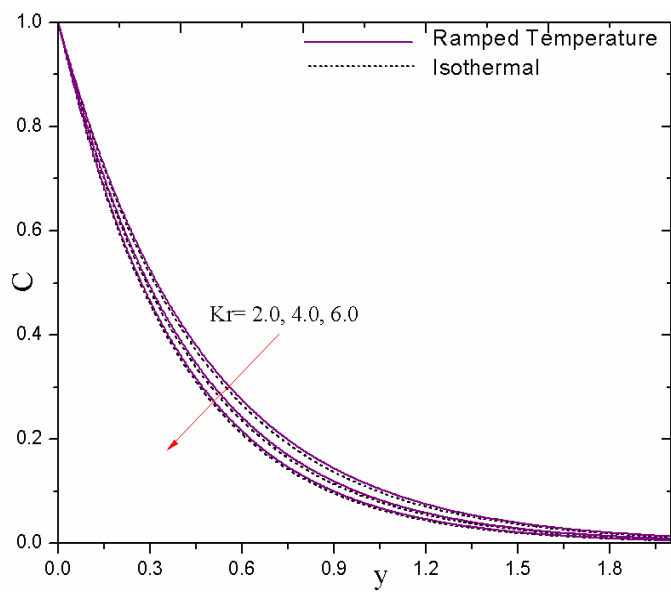

Figure 16. Concentration profile for chemical reaction

\section{CONCLUSIONS}

A computational solution has been carried out for investigating the thermal radiation effect on MHD casson fluid flow over an exponentially accelerated vertical plate in embedded porous medium with ramped wall temperature and ramped surface concentration. Using transformations a set of partial differential equations have been derived for the momentum, energy and species diffusion. Finite element method is engaged to solve the obtained equations. Our computational results have shown that:

a. Velocity profile accelerated with the increasing values of porosity parameter, thermal Grashof number, mass Grashof number, heat absorption, and radiation parameter. On the other hand cassion parameter, magnetic parameter, Prandtl number, Schmidt number and chemical reaction parameter have been shown to retard fluid velocity for both ramped and isothermal plates. 
b. Increasing the Prandtl number causes a deceleration in the temperature of the flow. While a reverse tendency has been identified against increasing values of heat absorption and radiation parameter for both ramped and isothermal plates.

c. Increasing Schmidt number and chemical reaction parameter results a reduction in concentration values for both ramped and isothermal plates.

\section{REFERENCES}

[1] S. Nadeem, R. U. Haq and C. Lee, "MHD flow of a Casson fluid over an exponentially shrinking sheet," Scientia Iranica, vol. 19, no. 6, pp. 1550-1553, 2012.

[2] S. A. Shehzad, T. Hayat, M. Qasim and S. Asghar, "Effects of mass transfer on MHD flow of casson fluid with chemical reaction and suction," Brazilian Journal of Chemical Engineering, vol. 30, no. 1, pp.187-195 2013.

[3] G. Makanda, S. Shaw and P. Sibanda, "Effects of radiation on MHD free convection of a Casson fluid from a horizontal circular cylinder with partial slip in non-Darcy porous medium with viscous dissipation," Boundary Value Problems, vol. 2015, no. 1, pp.1-14, 2015.

[4] J. Benazir, A Sivaraj and O. D Makinde, "Unsteady magnetohydrodynamic Casson fluid flow over a vertical cone and flat plate with non-uniform heat source/sink," Int. J. of Engineering Research in Africa, vol. 21, pp. 69-83, 2016.

[5] N. Ahmed, A. H. Sheikh and D. P. Barua, "Effect of fluid suction on an oscillatory MHD channel flow with heat transfer," Applications and Applied Mathematics: An International Journal, vol. 11, no. 1, pp. 266-284, 2016.

[6] M. Aziz, El. Abd and A. A. Afify, "Effect of Hall current on MHD slip flow of Casson nanofluid over a stretching sheet with zero nanoparticle mass flux," Thermophysics and Aeromechanics, vol. 26, no.3, pp 429-443, 2019.

[7] H. R. Kataria and R. P..Harshad, "Soret and heat generation effects on MHD Casson fluid flow past an oscillating vertical plate embedded through porous medium," Alexandria Engineering Journal, vol. 55, no.3, pp. 2125-2137, 2016.

[8] S. Mohyud-Din and I. Khan, "Nonlinear radiation effects on squeezing flow of a Casson fluid between parallel disks," Aerospace Science and Technology, vol. 48, pp.186-192, 2016.

[9] N. S. Akbar, "Influence of magnetic field on peristaltic flow of a Casson fluid in an asymmetric channel: Application in crude oil refinement," Journal of Magnetism and Magnetic Materials, vol. 378, pp. 463-468, 2015.

[10] K. Vajravelu, S. Sreenadh, R. H. Reddy and K. Murugesan, "Peristaltic transport of a Casson fluid in contact with a Newtonian fluid in a circular tube with permeable wall," International Journal of Fluid Mechanics Research, vol. 36, no. 3, pp. 244-254, 2009.

[11] P. Nagarani, G. Sarojamma and G. Jayaraman, "Exact analysis of unsteady convective diffusion in Casson fluid flow in an annulus-Application to catheterized artery," Acta Mechanica, vol. 187, no.1, pp. 189-202, 2006.

[12] S. Priyadharshini and R. Ponalagusamy, "Mathematical modelling for pulsatile flow of Casson fluid along with magnetic nanoparticles in a stenosed artery under external magnetic field and body acceleration," Neural Computing and Applications, vol. 31, no. 3,pp. 813-826, 2019.

[13] M. Sajid and T. Hayat, "Influence of thermal radiation on the boundary layer flow due to an exponentially stretching sheet," International Communications in Heat and Mass Transfer, vol. 35, no. 3, pp. 347-356, 2008.

[14] K. Bhattacharyya and G. C. Layek, "Effects of suction/blowing on steady boundary layer stagnation-point flow and heat transfer towards a shrinking sheet with thermal radiation," International Journal of Heat and Mass Transfer, vol. 54, no. 1-3, pp. 302-307, 2011.

[15] S. Pramanik, "Casson fluid flow and heat transfer past an exponentially porous stretching surface in presence of thermal radiation," Ain Shams Engineering Journal, vol. 5, no.1, pp. 205-212, 2014.

[16] A. M. RamiReddy, J. V. Reddy, N. Sandeep and V. Sugunamma, "Effect of nonlinear thermal radiation on MHD chemically reacting maxwell fluid flow past a linearly stretching sheet," Applications and Applied Mathematics: An International Journal, vol. 12, no. 1, pp. 259-274, 2017.

[17] Y-C. Han, T. F. Nie and Z. B. Yuan, "Mathematical and numerical analysis of radiative heat transfer in semi-transparent media," Applications of Mathematics, vol. 64, no. 1, pp. 75-100, 2019.

[18] S. R. Siva, A. J. Chamkha and A. K. Suram, "Heat and mass transfer effects on MHD natural convection flow past an impulsively moving vertical plate with ramped temperature," American Journal of Heat and Mass Transfer, vol. 3, no. 3, pp. 129-148, 2016.

[19] S. R. Siva and A. K. Suram, "Finite element analysis of heat and mass transfer past an impulsively moving vertical plate with ramped temperature," Journal of Applied Science and Engineering, vol. 19, no. 4, pp. 385-392, 2016.

[20] S. R. Siva, A. K. Suram and P. Modulgua, "Heat and mass transfer effects on MHD natural convection flow past an infinite inclined plate with ramped temperature," Journal of the Korean Society for Industrial and Applied Mathematics, vol. 20, no. 4, pp. 355-374, 2016.

[21] R. Nandkeolyar, M. Das and P. Sibanda, "Exact solutions of unsteady MHD free convection in a heat absorbing fluid flow past a flat plate with ramped wall temperature," Boundary Value Problems, vol. 247, no. 1, pp. 1-16, 2013.

[22] G. S. Seth, S. M. Hussain and S. Sarkar, "Hydromagnetic natural convection flow with heat and mass transfer of a chemically reacting and heat absorbing fluid past an accelerated moving vertical plate with ramped temperature and ramped surface concentration through a porous medium," Journal of the Egyptian Mathematical Society, vol, 23, no. 1, pp. 197-207, 2015. 
[23] Ch. Vijaya Bhaskar, S. R. Siva and A. K. Suram, "MHD natural convection flow past a moving verticalplate with ramped temperature," ARPN Journal of Engineering and Applied Sciences, vol. 13, no. 22, pp. 8846-8853, 2018.

[24] K. J. Bathe, "Finite element procedures," Prentice-Hall, New Jersey, 1996.

[25] J. N. Reddy, "An introduction to the finite element method, 3rd edition," McGraw-Hill Book Company, New York, 2006.

\section{BIOGRAPHIES OF AUTHORS}
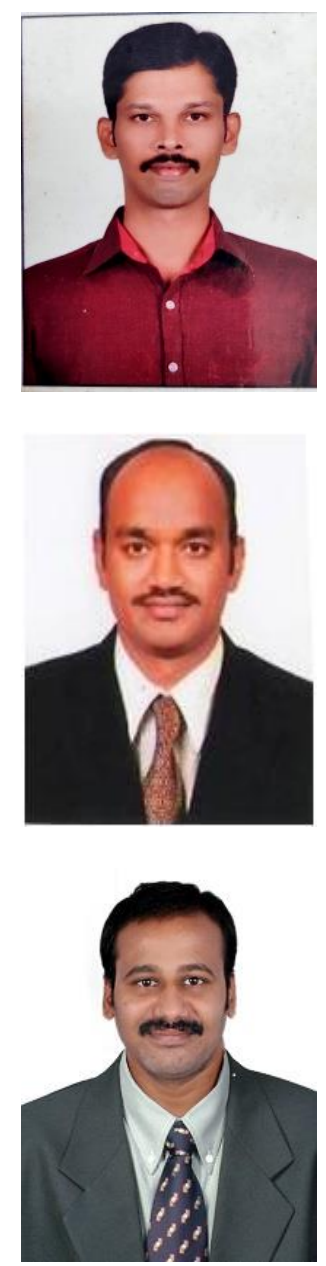

Mr. Vijayabhaskar Chathilla is an assistant professor in the Department of Engineering Mathematics, Vignan Institute of Technology and Science, Hyderabad, Telangana, India. He received his M.Sc in the mathematics from Osmania University, Hyderabad, India. He qualified in Telangana State Eligibility Test (TS-SET). He has more than thirteen years of experience of teaching and research. His current area of research studies includes fluid dynamics, magnetohydrodynamics, and heat- mass transfer. He has published research papers in reputed journals.

Dr Siva Reddy Sheri is an associate professor in the Department of Engineering Mathematics, GITAM University, Hyderabad Campus, Hyderabad, Andhra Pradesh, India. He received his Ph.D. in mathematics from Osmania University, Hyderabad, India. He has more than twenty years of experience of teaching and research. His current area of research studies includes fluid dynamics, magnetohydrodynamics and heat and mass transfer. He has completed major research project from Univerty Grants Commission (UGC) [F.No:42-22/2013 (SR) letter dated 12-032013]. He has published more than sixty research papers in reputable national/international journals.

Mr. Anjan Kumar Suram is an assistant professor in the Department of Engineering Mathematics, DRK college of Engineering and Technology, Hyderabad, Telangana, India. He received his M.Sc in mathematics from Osmania University, Hyderabad, India. He has more than thirteen years of experience of teaching and research. His current area of research studies includes fluid dynamics, magnetohydrodynamics and heat and mass transfer. He has published eight research papers in reputable national/international journals. 